\title{
Exigent Encounters with Vaccines Perplexes
}

\section{Sangeeta Kumari}

\author{
Amity Institute of Biotechnology, Amity University Haryana - 122413
}

\begin{abstract}
Background: The entire planet is now under significant threat of a terrible CoVid-19 epidemic Because of small clandestine organisms known as viruses. Only $1 \%$ of the population has been discovered, leaving the other $95 \%$ unexplored. The most difficult aspect of dealing with these minuscule organisms is their unpredictability, as viruses mutate quickly. Poliomyelitis, smallpox, measles, meningitis, and a few other deadly viral and bacterial diseases were successfully eradicated as a result of the immunisation push. The goal of this review article is to look at both the positive and negative aspects of vaccines. In order to improve the production, examine the quality of WHO vaccine recommendations in clinical practise guidelines (CPGs).

Methods: A systematic review of available microbial agent vaccines and pipelines (WHO, 2020) as well as the National Centre for Complementary and Integrative Health's websites was conducted. The US Department of Health and Human Services' Assessment of Guidance, Study, and Evaluation was compared to the Eligible WHO and FDA guidelines providing treatment and/or management recommendations. Approval stage for vaccination products. Food and Drug Administration, United States of America. Understanding vaccine research: How are AIDS vaccines tested? IAVI Article, first volume, 2003. WHO,1998 Recommendations for the quality control of 26 DNA vaccine volumes [17] Vaccines, 3rd Edition, Shock 12th Edition, and "History of Vaccination" in 111 volumes of proceedings are also recommended. Vaccines against almost 27 microbes are approved and ongoing research on vaccines against almost 130 microbial agents is ongoing (WHO, 2020; FDA, 2017). The decision to move to ART (Anti-Retroviral Treatment) regimens is based on the recommendations for clinical, immunological, and virological failure. Assay style output using DBS as opposed to plasma using a 1000 copies / $\mathrm{mL}$ viral load threshold ${ }^{[30]}$.

Conclusion: The high-scoring suggestions could be used as a foundation for future vaccine use in the context of various microbial illnesses in vaccine development. In addition to the graphical representation of the vaccine plan, various tools for designing guidance are being used to get insight.
\end{abstract}

Keywords: Vaccines, WHO Guidelines, Development stages, Challenges, COVID Vaccines

\section{INTRODUCTION}

"Vaccines are the tugboats of preventive health." - William Foege, Epidemiologist design the vaccine strategy to eliminate smallpox.

A vaccine, or an immunobiological agent meant to provide specific protection against disease, is an inactivated or attenuated pathogen or pathogen component (nucleic acid, protein) that confers immunity to the host and, when delivered to the host, triggers a defensive reaction in the cells ${ }^{[17]}$. In other words, the protection of susceptible persons from disease through the delivery of a living or modified agent (e.g., oral polio vaccine), suspension of killed species (as in pertussis), or inactivated toxin (as in tetanus). Immunization is defined as "the induction of active immunity in a susceptible host by introducing the pathogenic organism's specific antigen." Microbiology, immunology, epidemiology, public health, and pharmacy ideas are all included in vaccinology (Fig.1). Vaccines and vaccinations, on the other hand, are comparable.

Several vaccinations for numerous microbiological diseases were developed 
after the discovery of the smallpox vaccine. The primary research mission, as well as the production of vaccines, has evolved dramatically in the twenty-first century. Vaccinations against nearly 27 microbes have been approved, while research into vaccines against nearly 130 microbes is ongoing ${ }^{[33]}$. For more than two decades, it's been estimated that diseases currently treatable by licenced vaccines account for $20 \%$ of new-born mortality.
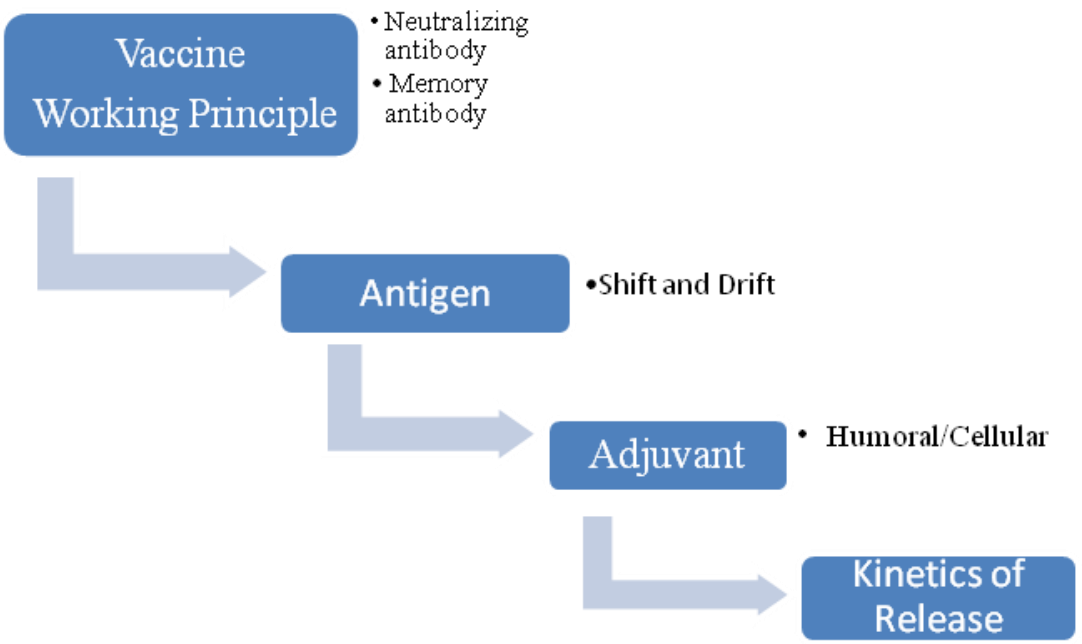

Fig. 1. Vaccine working principle

Vaccines are most effective in bacterial infections but are not a particularly profitable alternative for viral diseases for a variety of reasons. Coronavirus vaccines require a lot of study and stabilisation because it contains the largest sequence of RNA genomes (about 31,000 nucleotides). However, several research teams are developed few CoVid-19 vaccines already in market and administrated successfully

Available vaccines
- Cholera
- Dengue
- Diphtheria
- Hepatitis A
- Hepatitis B
- Hepatitis E
- Haemophilus influenzae type b (Hib)
- Human papillomavirus (HPV)
- Influenza
- Japanese encephalitis
- Malaria
- Measles
- Meningococcal meningitis
- Mumps
- Pertussis
- Pneumococcal disease
- Poliomyelitis
- Rabies
- Rotavirus
- Rubella
- Tetanus
- Tick-borne encephalitis
- Tuberculosis
- Typhoid
- Varicella
- Yellow Fever

among the population .Traditional procedures such as microbe attenuation and inactivation were the first steps toward modern vaccinology. ${ }^{[17]}$

The development of new technology such as glycoconjugates and novel vaccine adjuvants has recently altered the vaccination market. Microbial vaccines that are now available and in the pipeline are listed below in (Table-1): ${ }^{37]}$

Pipeline vaccines
- Pipeline vaccines
- Campylobacter jejuni
- Chagas Dise ase
- Chikungunya
- Dengue
- Enterotoxigenic Escherichia coli
- Enterovirus 71 (EV71)
- Group B Streptococcus (GBS)
- HerpesSimplex Virus
- HIV-1
- Human Hookworm Dise ase
- Leishmaniasis Disease
- Malaria
- Nipah Virus
- Nontyphoidal Salmonella Disease
- Norovirus
- Paratyphoid fever
- RespiratorySyncytial Virus (RSV)
- Schistosomiasis Dise ase
- Shigella
- Staphylococcus aureus
- Streptococcuspneumoniae
- Streptococcus pyrogenes
- Tuberculosis
- Universal InfluenzaVaccine
- CoronaVirus


We achieved significant milestones in terms of discovery and production during the evolutionary development of vaccines as described above. The first major shift occurred in 1995, when the first wholegenome sequencing of Hemophilus influenzae was completed, sparking the concept of "Reverse Vaccinology," a genome-based approach to vaccine production ${ }^{[4,19]}$. In 2016, researchers looked at reverse vaccinology in conjunction with

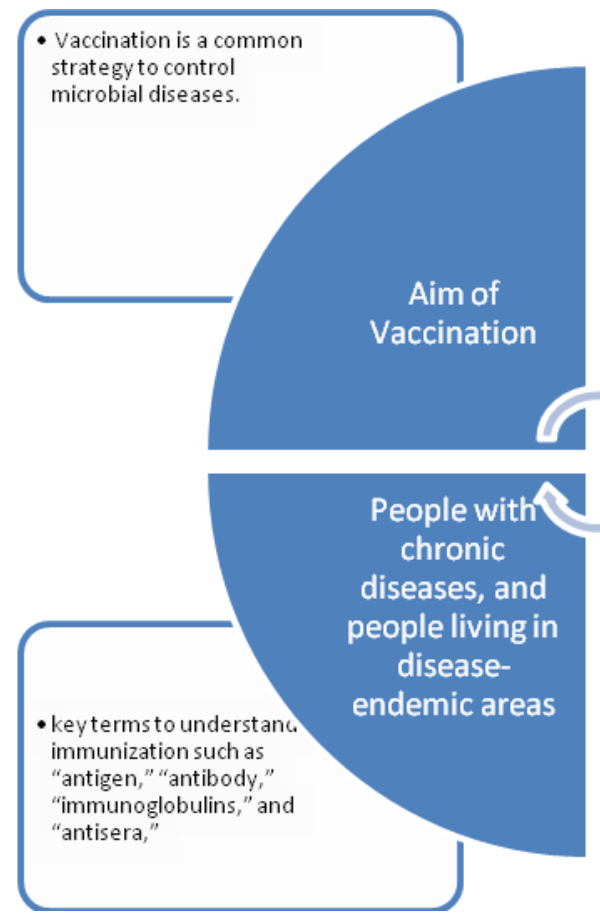

\subsection{Vigour of infection}

The infection rate is directly proportional to the probability about an infection. Infection tenacity is a complex mechanism directly related to transmission which can alter over a length of time. ${ }^{[15]}$

\subsection{Viral Load Threshold}

Anti-Retroviral Treatment (ART) is developed to overcome adherence risks and ensure whether ART regimens should be switched in the event of treatment failure (Table 2) ${ }^{[28]}$. Viral load testing is the best way to tell the difference between treatment failure and non-adherence (Table-3).Viral load can also serve as an idiom for both the person level and the likelihood of antibody characterisation of the human immune system at a single-cell level, which was a huge breakthrough. To quickly categorise highly immunogenic antigens, more specialised approaches are required to develop novel and more effective vaccine candidates. A diagram depicting the vaccine policy that was implemented to eradicate. In the diagram below, a schematic illustration of the vaccine policy used to eradicate dangerous illnesses is depicted (Fig.2.).

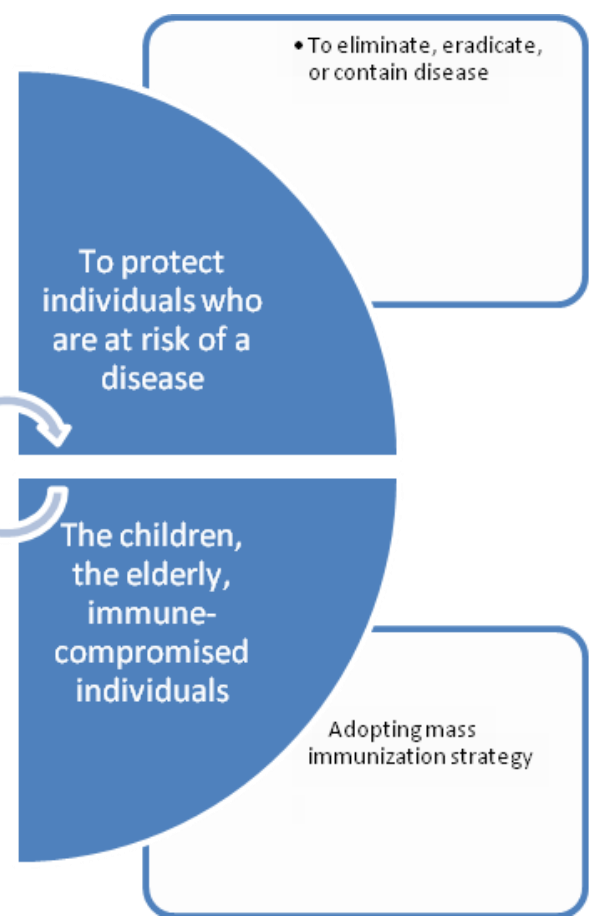

transmission and efficacy of preventive interventions (Fig. 3).

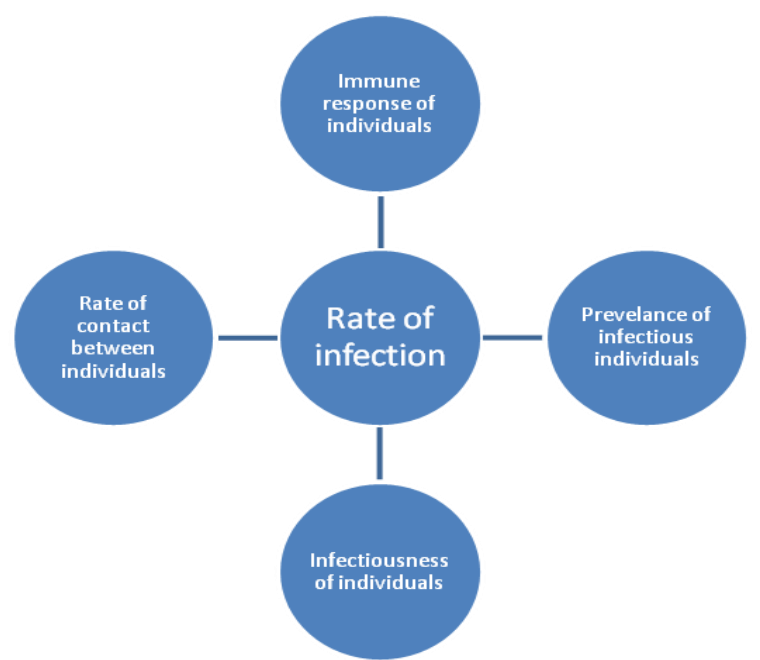

Fig. 3. The rate of infection depends upon transmission factors among individuals: 
Table-2. WHO 2017 Clinical, immunological, and virological failure guidelines for deciding to choose whether or not switch antiretroviral treatment regimens

\begin{tabular}{|c|c|c|}
\hline Failure & Definition & Comments \\
\hline Clinical failure & $\begin{array}{l}\text { Adults and adolescents } \\
\text { New or recurrent clinical event } \\
\text { indicating severe immunodeficiency } \\
\text { (WHO clinical stage } 4 \text { condition) } \\
\text { after } 6 \text { months of effective treatment } \\
\text { Children } \\
\text { New or recurrent clinical event } \\
\text { indicating advanced or severe } \\
\text { immunodeficiency (WHO clinical } \\
\text { stage } 3 \text { and } 4 \text { clinical condition with } \\
\text { the exception of TB) after } 6 \text { months of } \\
\text { effective treatment }\end{array}$ & $\begin{array}{l}\text { The condition must be differentiated } \\
\text { from immune reconstitution } \\
\text { inflammatory syndrome occurring } \\
\text { after initiating ART } \\
\text { For adults, certain WHO clinical stage } \\
3 \text { conditions (pulmonary TB and } \\
\text { severe bacterial infections) may also } \\
\text { indicate treatment failure }\end{array}$ \\
\hline $\begin{array}{l}\text { Immunological } \\
\text { failure }\end{array}$ & $\begin{array}{l}\text { Adults and adolescents } \\
\text { CD4 count at or below } 250 \text { cells } / \mathrm{mm}^{3} \\
\text { following clinical failure } \\
\text { or } \\
\text { Persistent CD } 4 \text { levels below } \\
100 \text { cells/mm } 3 \\
\text { Children } \\
\text { Younger than } 5 \text { years } \\
\text { Persistent CD } 4 \text { levels below } \\
200 \text { cells } / \mathrm{mm}^{3} \\
\text { Older than } 5 \text { years } \\
\text { Persistent CD } 4 \text { levels below } \\
100 \text { cells } / \mathrm{mm}^{3}\end{array}$ & $\begin{array}{l}\text { Without concomitant or recent } \\
\text { infection to cause a transient decline } \\
\text { in the CD4 cell count } \\
\text { Current WHO clinical and } \\
\text { immunological criteria have low } \\
\text { sensitivity and positive predictive } \\
\text { value for identifying individuals } \\
\text { with virological failure. There is } \\
\text { currently no proposed alternative } \\
\text { definition of treatment failure and } \\
\text { no validated alternative definition of } \\
\text { immunological failure }\end{array}$ \\
\hline $\begin{array}{l}\text { Virological } \\
\text { failure }\end{array}$ & $\begin{array}{l}\text { Viral load above } 1000 \text { copies/mL } \\
\text { based on two consecutive viral load } \\
\text { measurements in } 3 \text { months, with } \\
\text { adherence support following the first } \\
\text { viral load test }\end{array}$ & $\begin{array}{l}\text { An individual must be taking ART for } \\
\text { at least } 6 \text { months before it can be } \\
\text { determined that a regimen has failed }\end{array}$ \\
\hline
\end{tabular}

Table -3. Employing viral load threshold of 1000 copies/mL, the performance of assay types using DBS was compared to plasma WHO, 2017[34].

\begin{tabular}{|l|l|l|l|l|l|l|}
\hline Failure & $\begin{array}{l}\text { Abbott } \\
\text { RealTime }\end{array}$ & $\begin{array}{l}\text { Biocentric } \\
\text { Charge } \\
\text { Virale }\end{array}$ & $\begin{array}{l}\text { bioMerieux } \\
\text { Nucleisens }\end{array}$ & $\begin{array}{l}\text { Roche } \\
\text { TaqMan } \\
\text { FVE }\end{array}$ & $\begin{array}{l}\text { Roche } \\
\text { TaqMan } \\
\text { SPEX }\end{array}$ & $\begin{array}{l}\text { Siemens } \\
\text { kPCR }\end{array}$ \\
\hline $\begin{array}{l}\text { Sensitivity } \\
(95 \% \\
\text { confidence } \\
\text { interval }[\mathrm{Cl}])\end{array}$ & $\begin{array}{l}95 \% \\
(82-99 \%)\end{array}$ & $\begin{array}{l}95 \% \\
(71-99 \%)\end{array}$ & $\begin{array}{l}84 \% \\
(79-89 \%)\end{array}$ & $\begin{array}{l}85 \% \\
(77-91 \%)\end{array}$ & $\begin{array}{l}99 \% \\
(97-100 \%)\end{array}$ & $\begin{array}{l}91 \% \\
(69-98 \%)\end{array}$ \\
\hline $\begin{array}{l}\text { Specificity } \\
(95 \% \mathrm{CI})\end{array}$ & $\begin{array}{l}92 \% \\
(79-97 \%)\end{array}$ & $\begin{array}{l}55 \% \\
(35-74 \%)\end{array}$ & $\begin{array}{l}95 \% \\
(86-98 \%)\end{array}$ & $\begin{array}{l}94 \% \\
(85-98 \%)\end{array}$ & $\begin{array}{l}\text { (84\% } \\
(18-74 \%)\end{array}$ & $\begin{array}{l}88 \% \\
(75-94 \%)\end{array}$ \\
\hline
\end{tabular}

\section{Vaccine Major Types}

Vaccinations are divided into four categories: attenuated (live) vaccines, inactivated vaccines, toxoid vaccines, subunit vaccines, and conjugate vaccines (Fig . 4). To produce immunity, the initial vaccines for humans used weakened / attenuated viruses against viral infections that did not cause severe sickness (e.g., the early vaccination of smallpox produced from cowpox). The first human vaccine was the rabies vaccine (weakened / attenuated viruses were used). 


\section{Types of Vaccines}

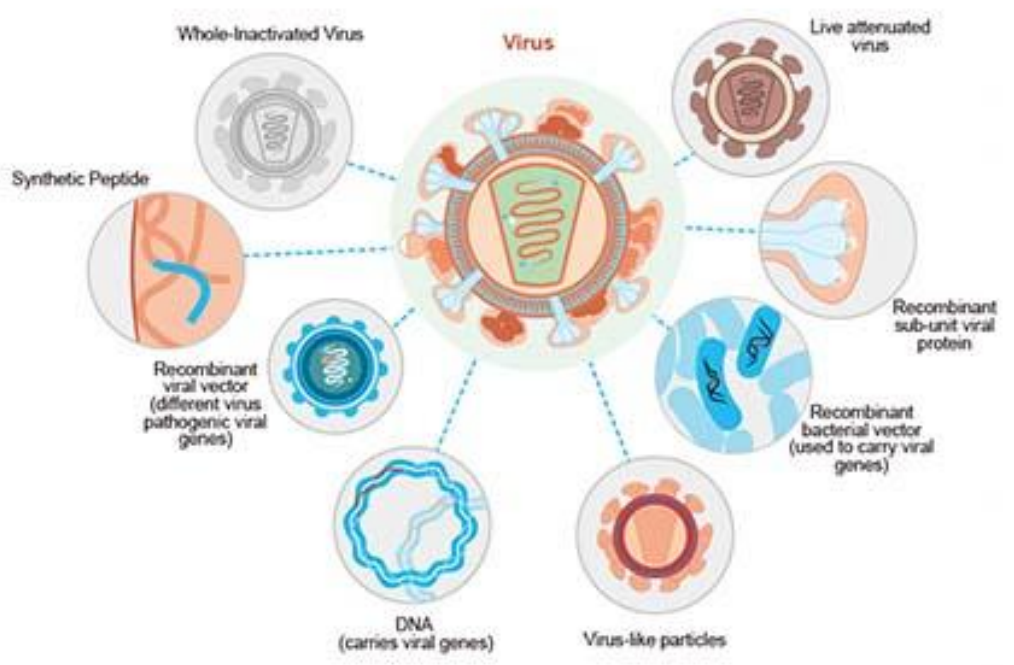

Live attenuated (LAV)

- Tuberculosis (BCG)

- Oral polio vaccine (OPV)

- Measles

- Rotavirus

- Yellow fever
Inactivated (killed antigen)

- Whole-cell pertussis (WP)

- Inactivated polio virus (IPV)

\section{Subunit (purified antigen)}

- Acellular pertussis (aP).

- Haemophilius influenzae type B(Hib),

- Pneumocoocal (PCV-7. PCV-10. PCV-13)

- Hepatitis B (HepB)

\section{Toxoid (inactivated toxins)}

- Tetanus toxoid (TI)

- Diphteria toxoid

Fig. 4. Types of Vaccines. Image Source: GenScript

The List of Vaccines According to Vaccine Type

\subsection{Live Attenuated Vaccines}

Live vaccines have been attenuated, or weakened is made using wild viruses or bacteria. After immunization, the weakened vaccine viruses or bacteria replicate (grow) in the vaccinated person to stimulate an immune response. There are many milestones attained during vaccine development (Fig. 5).

In vaccine recipients, live attenuated vaccines do not cause the disease. Only one dose is effective with live attenuated vaccines provided by injection. Oral vaccines, however, typically require three doses. Administration of a live attenuated vaccine may trigger serious illness due to uncontrolled replication (growth) of the vaccine virus if administered to an individual who has impaired immune system response (leukaemia or HIV infection, or is taking immunosuppressive medications). The rotavirus, chickenpox, measles, mumps, and rubella vaccinations are only a few examples of live attenuated vaccines. Live-attenuated vaccines are highly effective because of the physiological presentation of native antigen to the immune system of the host.
System and the earliest human vaccine that was developed by Serial Rabbit Virus Passages in Rabbits. In rather infrequent cases, the oral poliovirus vaccine, a single attenuated viral vaccine (OPV), mutations will accumulate as well as recombine with coinfecting other enteroviruses and returning to a pathogenic state. ${ }^{[11,14]}$

\subsection{Inactivated Vaccines}

Inactivated vaccines are composed of a fragment of microbial toxin, protein, or polysaccharide (sugar) using wild viruses or bacteria grown in a culture medium and inactivated prior to vaccination.

Vaccine antigens are unable to replicate (grow) or cause disease in the vaccinated organism. So, person with an impaired immune system response can safely be given this type of vaccine. A person's immunity faces a big obstacle to its efficacy. Normally, inactivated vaccines require several doses. In order to improve defence against disease, some inactivated vaccines may also require periodic supplementary doses. 


\subsection{Subunit Vaccines}

A subunit vaccine is a pathogen's surface protein, usually used to activate an immune response and induce acquired immunity to the pathogen it is extracted from. The inactivated vaccines for hepatitis A, influenza and polio are diphtheria, tetanus, pertussis, hepatitis $\mathrm{B}$, human papillomavirus, type $b$ Haemophilus influenzae, meningococcal and subunit vaccines for pneumococcal disease.

\subsection{Toxoid Vaccines}

Toxoid vaccines are prepared by adding harmless toxoids that activate the immune response to the toxin, thereby creating immunity. Toxoid is an inactivated harmful protein substance, the toxicity of which is suppressed by heat or chemical treatment, but the immunogenicity is preserved, i.e., the ability to produce an immune response. Tetanus, diphtheria, and pertussis toxins, for example, are inactivated in order to produce toxoids for use in Infanrix-Hexa, Infanrix-IPV, Boostrix-IPV, and ADT Booster Schedule vaccines.

\subsection{Polysaccharide Vaccines}

A part of the capsule which is invariably made of polysaccharide is used as an antigen in a Polysaccharide vaccine to activate the immune response. Polysaccharide (sugar) molecules, for example, are taken from the outer layer of serotypes of Streptococcus pneumoniae (pneumococcal) for use in the Pneumovax 23 specific group vaccine schedule.

\subsection{Conjugate Vaccines}

Polysaccharide (sugar) molecules are taken from the outer layer of encapsulated bacteria and the molecules are joined to the protein carrier by conjugate vaccines. Carrier proteins are membrane proteins that move molecules actively across a biological membrane. For eg, polysaccharide molecules of type $b$ (Hib) Haemophilus influenzae, serotypes of Streptococcus pneumoniae (pneumococcal) and one of four serogroups of Neisseria meningitidis (meningococcal) combined to carrier proteins for the Schedule vaccines Act-HIB, Prevenar 13, NeisVac-C, and Menactra respectively.

\subsection{Viral Vector- Based Vaccines}

Vaccine-based research since the 1980s has developed a number of viruses as vaccine vectors by engineering them to encode heterologous antigens that are shuttled by the vector into the host cells. In addition, immune responses against the related target pathogen are induced.

The use of live (replicating but sometimes attenuated) or non-replicating vectors is based on these vaccine technologies and represents a nifty platform that provides many advantages over others.

\subsection{Recombinant Vaccines}

The gene segment is inserted into the gene of another cell, such as a yeast cell, for a specific protein from the disease-causing organism to induce a protective immune response (protein of interest). It has the same form as the protein of interest when the cell replicates. For hepatitis B and human papillomavirus, yeast cells are used, such as Infanrix-hexa, HBvaxPRO, and Gardasil.

\subsection{DNA Vaccines}

As early as the 1990s, research on DNA vaccines began, it is a plasmid containing the DNA sequence that encodes the antigen against a particular characteristic immune response and relies on the in-situ development of the target antigen that is further directly added to the tissue ${ }^{[31]}$. This approach provides many possible advantages over conventional methods, including activation of the responses of both $\mathrm{B}$ and $\mathrm{T}$ cells, increased stability of the vaccine, lack of any infectious agent, and the relative ease of large-scale manufacture. The plasmid contains unique genetic material that helps the Escherichia coli vector to spread and select. Similarly, stable vector-based replication inheritance that supports high plasmid yields during 
bacterial growth and a selectable marker, mainly the gene for bacterial antibiotic resistance against Kanamycin. In the new generations of DNA vaccines, because regulatory safety concerns are raised against the prevalence of non-functional sequences, in specific antibiotic resistance markers, for human use, the marker has been substituted or modified. The semi-synthetic mini-circle DNA (devoid of bacteria-derived plasmid DNA) and the fully synthetic DoggyboneTM (Walter et al.,2014) have been generated in recent years. The eukaryotic cell expression cassette consists of a 5 'cytomegalovirus (CMV) promoter that supports high levels of transcription, the gene of interest, and a 3' polyadenylation (poly A) signal needed for nuclear export from the genes of rabbit $\beta$-globin or bovine growth hormone. Intramuscular (IM) or intradermal (ID) injection using a standard needle is the most common route of administration. Vaccination with a DNA vector alone, however, generally results in significantly low immunogenicity, especially in large animal and human models (Wang,2004). The need for DNA vaccines to cross two cellular membranes to achieve protein expression is plasma, as well as the nuclear membrane factor that can play major part. These involve numerous delivery devices such as gene arms, needle-free injection devices (jet injection) and in vivo electroporation, which are among the most commonly used in preclinical and clinical trials and have been shown to produce promising results. Different DNA encapsulation formulations containing cationic lipids and cholesterol in lipid nanoparticles, adsorption to polymers such as polyethyleneimine and adsorption or encapsulation of biodegradable nanoparticles, such as poly (lactic-coglycolic acid) (PLGA) or chitosan have been tested. These techniques improve the DNA molecule's absorption into the cell and thus enhance the expression of antigen. In addition, numerous methods have been developed to alter and enhance DNA mediated immune responses. Molecular adjuvants such as pattern recognition receptor (PRR) ligands and various cytokines, most commonly IL-12, have been identified ${ }^{[12]}$ and are co-delivered with the encoded antigen and strategies to guide the antigen to some precisely targeted antigen presenting cells (APCs) to enhance immune responses. In tandem with other vaccine innovations, such as protein- or viral vectorbased vaccines, DNA vaccines have also been effectively used for prime-boost formulations.

\subsection{RNA Vaccines}

The mRNA throughout the vaccinated subject is an intermediate genetic information provider used as a template for the manufacturing of endogenous proteins. As prophylactic vaccines against pathogens that also cause infectious diseases, two major types of RNA are used:

a) Non-replicate mRNA

(b) Self-amplifying mRNA Amplifier

The sequence of the antigen of choice, flanked by $5^{\prime}$ and $3^{\prime}$ untranslated regions (UTRs), contains non-replicating mRNA. Compared to the potential of using non-replicating mRNA vaccines, Schalke mentioned the advantages of just using Vaccines are rooted in the simplicity of the build, the small size of the RNA, and the lack of any additional encoded proteins that might cause unwanted immune responses relative to self-amplifying mRNA. ${ }^{[7]}$ Pardi researched the efficacy of translated mRNA in context to the vaccine use. It's in vitro transcription of a cDNA template, usually plasmid DNA (pDNA) developed in E, generates non-replicating mRNA ${ }^{[9]}$. Coli.32 Employing restriction enzymes, the pDNA template is formulated and transcribed in vitro into mRNA in a mixture containing DNA-dependent recombinant phage RNA polymerase (typically derived from phage T7 or T3 or Sp6) and nucleoside triphosphates (NTPs). After purification, Weissman purified single mRNA product is obtained via FPLC or HPLC. ${ }^{[29]}$. In target cells transcribed immunogenic mRNA by up to 1,000-fold increased protein output in 
primary human DCs transfected with purified HPLC compared to unpurified mRNA. ${ }^{[9]}$ The transcribed mRNA product contains an open reading frame (ORF) protein-encoding flanked by crucial components for the reading (ORF). ${ }^{[5,16]}$ The 5 'cap is important in the development of stable mature mRNA and increases protein expression by binding to the initiation factor $4 \mathrm{E}$ of eukaryotic translation. ${ }^{[18]}$ The 5' cap can be incorporated during transcription by integrating an analogue or anti-reverse cap (ARCA) further into reaction or subsequently using the capping complex of the vaccinia virus. ${ }^{[10]}$ The half-life and consistency of eukaryotic or viral UTRs are higher than for the expression of mRNA 32. [09,27] The poly A tail of an optimal length is a significant signalling component to enhance translation and can be encoded into the DNA template or enzymatically incorporated through transcription. ${ }^{[35]}$ he ORF sequence can be optimised by enriching the GC content using. ${ }^{[23]}$ or by substitute uncommon codons of frequently used synonymous codons which lead to increased protein Chemically modified nucleosides can minimize innate effectiveness of the immune system as well as increase the translation of the mRNA. ${ }^{[9]}$

Self-amplifying mRNA vaccines are primarily based on the genome of a alphavirus, from which the structural protein-encoding genes have been replaced mostly with antigen of choice. The viral RNA is processed and transcribed by the viral RNA polymerase despite some gene deletions. Self-replicating with certain serious challenges over non-replicating mRNA vaccines that, as a consequence of the large size of these vaccines, the lower yields and increased occurrence of abortive constructs pose challenges to vaccine production ${ }^{[9]}$. The additional mRNA contains a sub-genomic promoter and a large ORF encoding for non-structural proteins which are deciphered into four functional components (nsP1, nsP2, nsP3, and nsp4) following delivery of the vaccine further into cytosol by the encoded RNA- dependent RNA polymerase. (RDRP). ${ }^{[7]} \mathrm{A}$ negative-sense copy of the genome which serves as a framework for two positivestrand RNA molecules is generated by RDRP: the genomic mRNA and the shorter sub-genomic mRNA. At very elevated levels, this sub-genomic mRNA is transcribed, leading the mRNA amplification to encrypt the antigen of choice. Any genetic information encoded by the self-amplifying mRNA vaccine can thus be amplified multiple times, resulting in high levels of expression of antigen through relatively low doses of the vaccine, which is a vaccine. Further appealing attribute of self-amplifying mRNA vaccines compared to non-replicating mRNA vaccines LNPformulated self-amplifying mRNA encoding firefly luciferase induced protein expression lasting nearly two months following injection of IM in mice. [5] Whereas luciferase expression from protamineformulated, non-replicating ID administered by mRNA was typically only detected for a few days . Although further investigation is necessary for possible interactions between the host and the coded alpha viral nonstructural proteins.

Self-amplifying mRNA studied by Lundstrom is most frequently supplied with synthetic delivery vehicles, and an alternative method is to package and transport virus-like replicon particles (VRPs) developed by a helper cell line that provides trans capsid and glycoprotein genes. Though absence of structural protein genes found in VRPs prevents the production of additional viral particles and the dissemination of cells to cells, VRPs are capable of infecting cells and of expressing in vitro and in vitro the antigen of choice. While both preclinical and clinical results for the VRPs are promising, during the production process, this technology involves the use of electroporation of the genetic material into cell culture cells. Although electroporation has been successfully used on a scale sufficient to provide material for a phase I study under GMP conditions, costeffective industrial-scale production may be 
challenging. In addition, there are some safety issues associated with VRPs, because recombination. During their development in cells containing both replicon and helper RNAs, or co-packaging of replicon and helper RNAs VRPs could perhaps lead to the generation of infectious viruses. Exogenous mRNA has to enter the cytoplasm during the delivery of mRNA vaccines, where protein expression can take place. The plasma or endosomal lipid membrane in this stage provides a barrier to be crossed as effectively as possible by the mRNA vaccine. The initiation of an efficient immune response entails stimulation by the mRNA vaccine of the innate immune system. Consequently, many methods have been developed for enhancing both cell delivery and adjutancy segment of mRNA vaccines.

\section{The Development of Modern Vaccines 3.1. By Reverse Vaccinology}

In 2016 the "reverse vaccinology" entered the stage an emerging new technologies in the field of vaccines such as glycoconjugates and the introduction of novel vaccine adjuvants changed, however the biggest change came with the first sequencing of the Heamophilus influenzae whole genome in 1995, a discovery that allowed the birth of "Reverse Vaccinology," a genome-based approach to vaccine development. ${ }^{[20,4]}$ Further analysed the Neisseria meningitidis serogroup B strain whole genome, identified as novel candidates as well as the development of a four-component meningococcus $B$ vaccine (4CMenB). [26] This recently licensed vaccine has already shown incredible effectiveness in the UK with $82.9 \%$ protection against all MenB strains in infants. The vaccine development further evolved moved forward with the advancement of new methodologies and technological breakthroughs. The human immune system is analyzed at a single cell level allowing the characterization of the antibody response like never before. ${ }^{[19]}$

\subsection{Use of Peptide-Antigen Derived for Germline Targeting Vaccinology}

Th germline-targeting (GT) antigens for vaccine development are another pivotal example that underlies the outstanding potential of reverse vaccinology. Certainly, the combined knowledge acquired by the identification and characterization of novel antigens plus the functional/genetic analysis of human monoclonal antibodies naturally produced by infected or vaccinated human donors, can be used to design antigenderived peptides, capable of tailoring the antibody immune response. The whole antigen set use can result in a strain specific response. The development of GT-antigens can lead to the elicitation of broadly neutralizing antibodies (bnAbs) capable of clearing multiple infective strains in case of highly variable pathogens such as HIV.

The two-step approach using different rationally designed immunogens as:

(1) The germline precursor B cell of antibodies possesses broadly neutralizing activity;

(2) Guiding the bnAb population by driving their maturation affinity toward the highly immunogenic epitope of interest. To elicit a specific class of HIV-1 gp120 CD4-binding site specific-bnAbs known as VRC01, through the use of engineered outer domain germline-targeting (eOD-GT) peptides. The interest to primeVRC01-bnAbs arises from their ability to mimic the CD4-binding to the gp120 receptor binding site and their capability to potently neutralize (median IC50 40ng/mL) up to $98 \%$ of a large panel of global HIV-1 isolates. ${ }^{[21]}$ An in-depth analysis of the VRC01 genetic features has shown peculiarities in this class of bnAbs. These analyses were paramount for the development of novel and potentially therapeutic candidates to fight HIV infections. Few examples of the use of VRC01-bnAbs as a therapeutic tool are the monoclonal antibodies named VRCHIVMAB060-00-AB(VRC01) and a FC modified version of this latter named VRC01LS. Currently two bnAbs 
(NCT02568215, NCT02716675, and in reducing acquisition of HIV-1 infection. NCT02599896) are under clinical investigation evaluating safety and efficacy

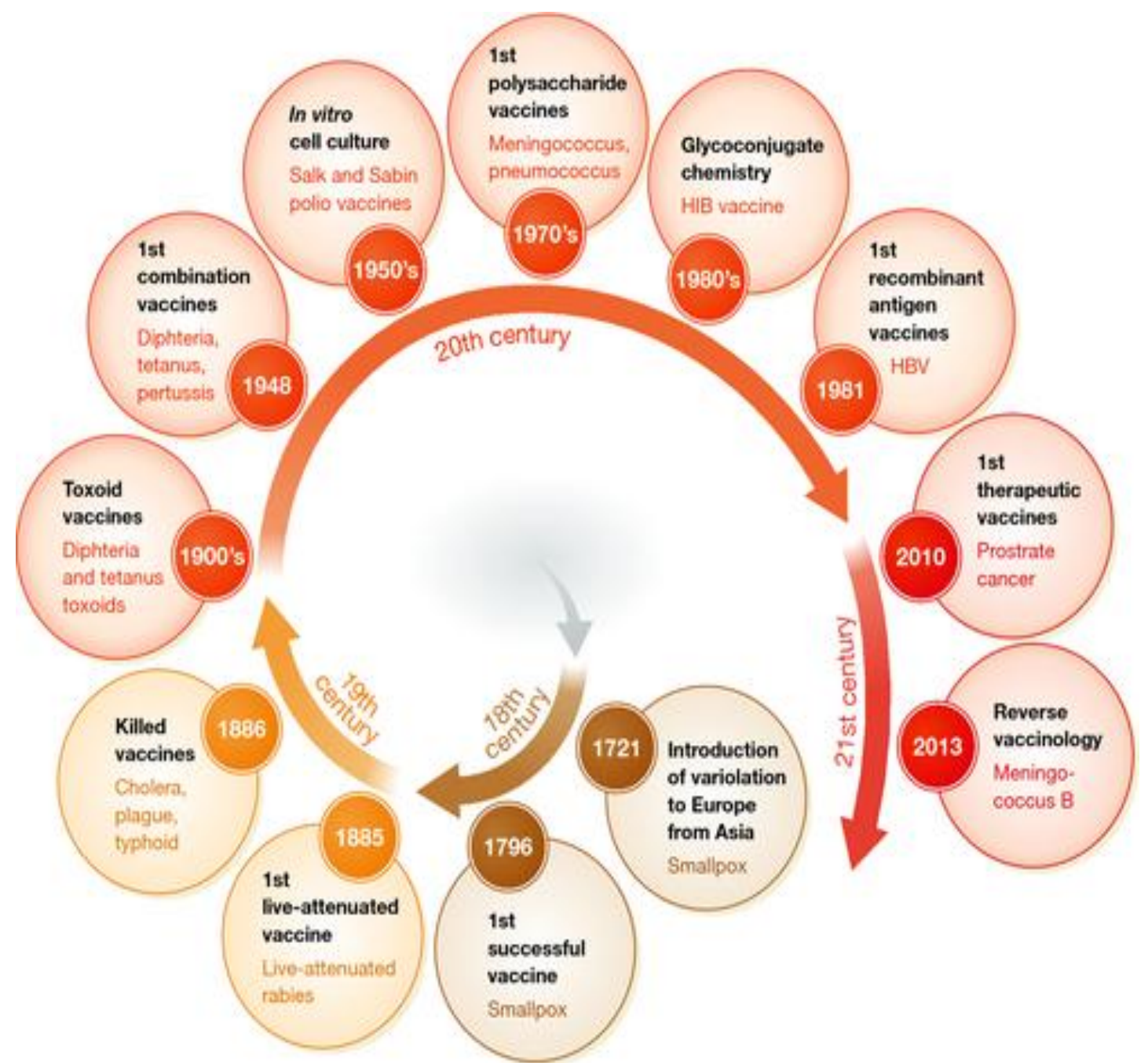

Fig. 5. Major milestones development of vaccinology and vaccine design. [2]

\section{Stages of Vaccine Development}

The general stages of the development cycle of a vaccine are according to WHO Guidelines. ${ }^{[3]}$

- Exploratory stage

- Pre-clinical stage

- Clinical development

- Regulatory review and approval

- Manufacturing

- Quality control

Clinical development is a threephase process. During Phase I, small groups of people receive the trial vaccine. In Phase II, the clinical study is expanded and vaccine is given to people who have characteristics (such as age and physical health) similar to those for whom the new vaccine is intended. In Phase III, the vaccine is given to thousands of people and tested for efficacy and safety.

Many vaccines undergo Phase IV formal, ongoing studies after the vaccine is approved and licensed (Fig. 6.). 


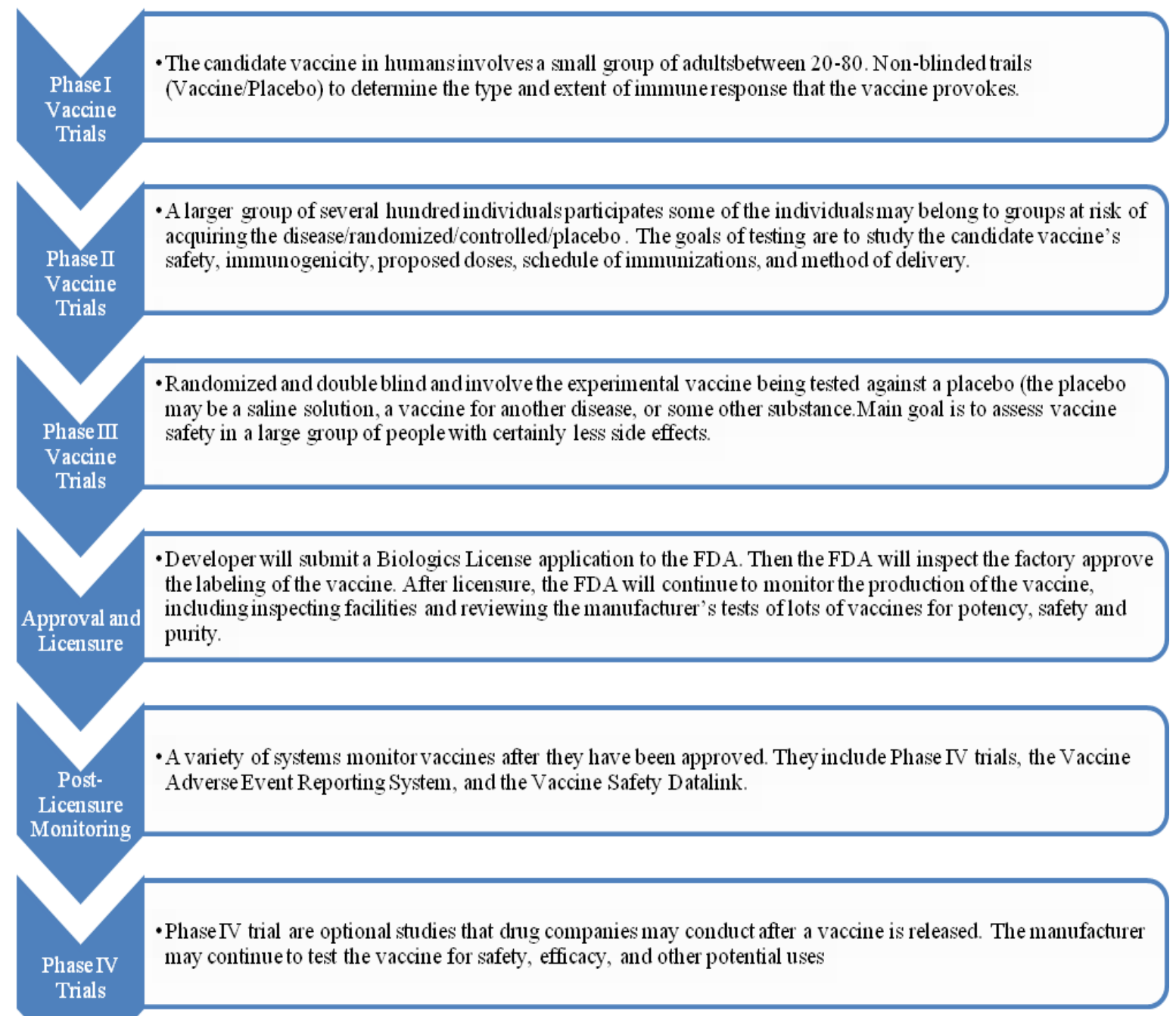

Fig. 6. Next Steps: Clinical Studies with Human Subjects.

Sources: U.S Department of Health and Human Services. Vaccine product approval process. U.S.

VAX. Understanding vaccine trials: How are AIDS vaccines tested? IAVI Report. Volume 1, no. 1. August $2003^{[3]}$

Plotkin, S.A., Orenstein, W.A., Offit, P.A., eds. Vaccines, 5th ed. Philadelphia: Saunders, 2008. Chapters 3 and 73.Table above from The Children's Vaccine Initiative: Achieving the Vision. National Academies Press. ${ }^{[17]}$

\section{VAERS (The Vaccine Adverse Event Reporting System)}

The Vaccine Adverse Event Reporting System was established_by CDC and FDA_in 1990. The main purpose of formation is "to detect possible signals of adverse events associated with vaccines." Around 30,000 events are reported each year to VAERS. Between $10 \%$ and $15 \%$ of these reports describe serious medical events that result in hospitalization, lifethreatening illness, disability, or death. ${ }^{[24]}$

VAERS is a voluntary reporting system. An association or disparity between a vaccination and an adverse event may report that event and information about it to VAERS from a health care provider and family/ friend of the patient. The CDC then investigates the event and the adverse event was caused by the vaccination.

The CDC states that they monitor VAERS data to:

- Detect new, unusual, or rare vaccine adverse events

- Monitor increases in known adverse events 
- Identify potential patient risk factors for particular types of adverse events

- Identify vaccine lots with increased numbers or types of reported adverse events

- Assess the safety of newly licensed vaccines

Not all adverse events reported to VAERS are caused by a vaccination. The two occurrences may be related in time only. The CDC states that many adverse events such as swelling at the injection site are underreported. ${ }^{[25]}$

VAERS has successfully identified several rare adverse events related to vaccination. Among them are

- An intestinal problem after the first vaccine for rotavirus was introduced in 1999

- Neurologic and gastrointestinal diseases related to yellow fever vaccine
- According to ${ }^{[17]}$ VAERS identified a need for further investigation of MMR association with a blood clotting disorder, encephalopathy after MMR, and syncope after immunization.

\section{Vaccine Efficacy and Effectiveness}

Vaccine efficacy has its effect on both individual and population levels. The "population level effects" of vaccination depend on the coverage and distribution of the vaccines.

"Biological or individual level effect" of vaccine includes effects on susceptibility (VEs) on infectiousness (VEi) and disease progression (VEp).

In (Fig 7) showing the types of population effect of vaccination. The public health effect of vaccination programs depends on the effect of both vaccination and the unvaccinated population. ${ }^{[15]}$.

\section{Indirect effect}

The population level effect of widespread vaccination on people not receiving vaccine

\section{Total effect}

Combination of population level effect and effect of vaccination on individuals receiving vaccine

Overall public health effect
The effect of vaccination program based upon weighted average of indirect effect on the individual not receiving vaccine and total effect on individual receiving vaccination.
'Efficacy of vaccine' is the percentage reduction in the incidence of disease due to vaccination determined using the following equation:

$\mathrm{VE}$ (percent) $=(\mathrm{RU}-\mathrm{RV}) / \mathrm{RU}=100$ (percent)

$\mathrm{RU}=$ the probability of incidence or attack rate in unvaccinated individuals and $\mathrm{RV}=$ the rate of incidence or attack in vaccinated individuals.

It is possible to reformulate vaccine efficacy as:

$\mathrm{VE}=1-\mathrm{RV} / \mathrm{RU}>100$
Where in vaccinated and unvaccinated persons, RV / RU is the relative risk or rate ratio.

Field conditions for observational trials within a vaccine programme or assessed by research performed under standard programme conditions. The vaccine effectiveness of many vaccines such as Measles is $90-95$ percent; mumps: $72-88$ percent; and rubella 95-98 percent. Among other items, like protection, there is an evaluation of the vaccine's effectiveness in vaccine trials. ${ }^{[8]}$ This is an important criterion for the licensing of vaccines and 
for programmatic use decisions. For example, the effectiveness of the measles vaccine depends on the existence of inhibitory maternal antibodies, the immunological maturity of the vaccine recipient, and the dose and strain of the vaccine virus. ${ }^{[28]}$ The efficacy of the vaccine depends on internal or individual variables.

\section{Recent Vaccine development under new challenge of pandemic COVID 19}

The global initiative to create and distribute an effective vaccine for the COVID-19 coronavirus disease has yielded some promising options a year after the pandemic began. The manufacture of multiple vaccines at such a rapid pace is unprecedented; usually, the process takes eight to fifteen years. Immunization of the population by vaccination is regarded as a public health priority in the current context of the SARS-COV-2 pandemic. The genetic sequencing of SARS-COV-2 was completed in less than a month. Since then, scientists have been working around the world to develop a vaccine. Presently, vaccinating a sufficient number of the world's populationwhich is imperative for bringing the pandemic under control- is facing a fresh set of obstacles, including dangerous new strains of the virus, global rivalry for a small supply of doses, and public scepticism about the vaccines. The majority of vaccines that have been licensed for restricted use were produced by companies and research groups in China, Russia, and the United States. In March 2020, the first clinical trial in the United States started in Seattle with a vaccine developed by Moderna Inc. After it appeared to be highly effective, the vaccine was approved for emergency use in the United States, members of the European Union, and many other countries. In largescale trials, it appeared to be very successful. Following similar promising results, regulatory agencies in dozens of countries approved a vaccine developed by Pfizer and BioNTech. The vaccine from Johnson \& Johnson was set to hit the market in February 2021, but it was halted in April in the United States and elsewhere due to exceptionally unusual cases of blood clotting. Several other COVID-19 vaccine candidates are undergoing large-scale clinical trials, and pharmaceutical firms, research institutions, and government agencies are developing about 180 potential vaccines in preclinical stages (Table-4).

Table-4 Potential Vaccine available for COVID 19 immunization drive.

\begin{tabular}{|c|c|c|c|c|c|c|}
\hline \multicolumn{7}{|l|}{ COVID-19 Vaccines } \\
\hline Manufacturer/Institute & Country & $\begin{array}{l}\text { Clinical } \\
\text { Trails }\end{array}$ & Efficacy & Doses & $\begin{array}{l}\text { Approved in at } \\
\text { least one country }\end{array}$ & $\begin{array}{l}\text { Approved in } \\
\text { United States }\end{array}$ \\
\hline Gamaleya Research Institute & Russia & 3 & $91 \%$ & 2 & Yes & \\
\hline CanSino & China & 3 & $66 \%$ & 1 & Yes & \\
\hline Sinopharm (Beijing) & China & 3 & $79 \%$ & 2 & Yes & \\
\hline Sinopharm (Wuhan) & China & 3 & $82 \%$ & 2 & Yes & \\
\hline Sinovac & China & 3 & $\begin{array}{r}50 \%- \\
91 \%\end{array}$ & 2 & Yes & \\
\hline $\begin{array}{l}\text { Central drugs control organization- } \\
\text { Serum Institute of India }\end{array}$ & India & 3 & $80 \%$ & 2 & Yes & \\
\hline Bharat Biotech & India & 3 & $78 \%$ & 2 & Yes & \\
\hline State Vector Institute & Russia & 3 & $92 \%$ & 2 & Yes & \\
\hline Oxford-AstraZeneca & $\begin{array}{ll}\text { United } & \text { Kingdom, } \\
\text { Sweden } & \end{array}$ & 2 and 3 & $79 \%$ & 2 & Yes & \\
\hline Pfizer-BioNTech & $\begin{array}{l}\text { United } \quad \text { States, } \\
\text { Germany }\end{array}$ & 2 and 3 & $91 \%$ & 2 & Yes & Yes \\
\hline Johnson \& Johnson & United States & 3 & $\begin{array}{r}64 \%- \\
72 \% \\
\end{array}$ & 1 & Yes & Yes \\
\hline Moderna & United States & 3 & $>90 \%$ & 2 & Yes & Yes \\
\hline Novavax & United States & 3 & $\begin{array}{r}49 \%- \\
96 \% \\
\end{array}$ & 2 & & \\
\hline
\end{tabular}

\section{DISCUSSION}

7.1.Future Prospects
Vaccine manufacturing requires major investment in advanced technology 
with a well-equipped production plant and the establishment of teams with multidisciplinary expertise in the large-scale production of biological products. The most challenging gaps to fill are that of identifying biomarkers. A prompt goal line we should set is to exploit the trials in the attempt to identify signatures of vaccine efficacy that can guide the early selection of the most promising vaccine candidates for the future. Considering the desirable responses and necessary for protection can be induced by a vaccine that will unlock the door to rationally designing effective nextgeneration vaccines. Identify biomarkers that correlative with vaccine protection or safety.

To identify novel protective epitopes by the use of antibody repertoire analysis. Further the use structural information on protective epitopes to design better immunogens. Rally knowledge of hostpathogen interactions and mechanisms of protection. Capture synergy of using different adjuvants or multiple vaccine technologies. Main emphasis on the safety concern for elderly, pregnant women, and infants during development of dedicated vaccines. Further studies will be based on the impact of vaccines in combination with antibody-based immunotherapy.

In the Indian context, the prominent role in the vaccine manufacturing Serum institute of India came out with a $\$ 1$ vaccine.

I sum-up this review with the famous quote of Dr Jonas Salk an American physician, medical researcher and virologist who developed one of the first successful polio vaccines.

\subsection{Challenges}

Due to genomic inconsistencies of the virus, they have a great tendency to mutate easily. Another issue is the instability of its constituent and immune response of the person. As viruses have the smartest machinery, they can easily hijack the host cell's nucleic acid and protein synthesis activity. So that more copies of the virus can be made and further manipulate its biochemical mechanism following the environment and the dynamic changes in their protein envelope. Made up of singlestranded ribonucleic acid (RNA), this genetic material exists on a single strand, unlike a double-stranded DNA. Once inside the human body, it easily breaks and rearranges itself thus enabling viruses to mutate quickly, meaning that any cure or vaccine made for a specific RNA may quickly become obsolete as the antibodies released by vaccines cannot hit the specific target virus. So, the vaccines are not very efficacious against various viral diseases.

Vaccine development requires solid aptitude and rigorous research and development set up. Furthermore, a rigorous study is required to understand the nature of host and pathogen interaction. Perplexities of vaccine described by Hopps are potency variation, stability problems, imbalance of microbial components, virulence factor, microbial / chemical contamination issues. Vaccines of SERS, MERS, AIDS, Influenza, Ebola, and many other diseases are still in the nascent stage of headway.

"The reward for work well done is the opportunity to do more."

\subsection{Inference}

Pandemics such as Covid-19, HIV, Ebola, and Zika have raised the awareness of global threats to human health posed by known as well as newly emerging pathogens and can provide the impetus to prepare against future pandemics by promoting the development of vaccine platforms that can tackle the challenges of outbreak situations. The prerequisites representing highly versatile technologies that provide solutions for fast vaccine manufacturing to some extent subjugate these challenges. New platforms, such as viral vector and nucleic acid-based vaccines meet each vaccine technology has its advantages and disadvantages related to its ability to induce certain immune responses, manufacturing capacity, and safety for human use. In summary, the paradigm shift of innovative 
technologies in the last century has allowed the various avenues for the development of novel vaccines targeting new diseases or new target populations.

\section{ACKNOWLEDGMENTS}

The authors thanks to Amity University to provide all support.

\section{Author contributions (Single Author)}

Conceptualization: SK. Methodology: SK. Writing - Original Draft: SK . Writing - SK Review \& Editing: SK.

\section{Conflict of Interest}

The authors declare that they have no competing interests.

\section{Funding} any funding.

This review article did not require

\section{Ethical Statement}

This research/review article did not require ethical approval as it does not involve any human or animal experiments.

\section{Supplementary Material}

The following are Supplementary material to this article:

For more information

GIVS 2006-2015 at

http://www.who.int/immunization/givs/en/

UNIAIDS Global Report at

http://www.unaids.org/en

WHO report 2010: http://www.who.int

HVTN505 result update at
http://www.hvtn.org/505-announcement25April2013.html

WHO table of vaccines: http://www.who.int/vaccine_research/links/ Rainbow/en/index.html

WHO:

http://www.who.int/malaria/world_malaria_ report_2011/en

\section{REFERENCES}

1. Cohen YZ, Caskey M. Broadly neutralizing antibodies for treatment and prevention of HIV-
1infection.CurrOpinHIVAIDS.2018;13:366

-73.doi: 10.1097/COH.0000000000000475

2. Delany, I., Rappuoli, R., \& De Gregorio, E. (2014b). Vaccines for the 21st century. EMBO Molecular Medicine, 6(6), 708-720. https://doi.org/10.1002/emmm.201403876

3. Esparza, J. et al. Estimation of "needs" and "probable uptake" for HIV/AIDS preventive vaccines based on possible policies and likely acceptance (a WHO/UNAIDS/IAVI study). Vaccine, 2003. 21(17-18), 20322041. $\quad$ https://doi.org/10.1016/s0264410x $(02) 00775-2$

4. Fleischmann RD, Adams MD, White O, Clayton RA, Kirkness EF, Kerlavage AR, et al. Whole-genome random sequencing and assembly of Haemophilus influenzae Rd. Science. 1995; 269:496-512. doi: $10.1126 /$ science. 7542800

5. Geall AJ, Verma A, Otten GR, Shaw CA, Hekele A, Banerjee K.Nonviral delivery of self-amplifying RNA vaccines. Proc Natl Acad Sci USA.2012;109:14604-9. doi: 10.1073/pnas.1209367109

6. Halloran, M. E., Struchiner, C. J., \& Longini, I. M. (1997b). Study Designs for Evaluating Different Efficacy and Effectiveness Aspects of Vaccines. American Journal of Epidemiology, 146(10), 789-803. https://doi.org/10.1093/oxfordjournals.aje.a 009196

7. Iavarone C, O'hagan DT, Yu D, Delahaye NF, Ulmer JB. Mechanism of action of mRNA-based vaccines. Expert Rev Vaccines (2017) 16:871-81. doi: 10.1080/14760584.2017.1355245

8. Jamuna, P. (2010). Evaluation of certain food additives. Sixty-ninth report of the Joint FAO/WHO Expert Committee on Food Additives (JECFA). WHO Technical Report Series No. 952. 2009. World Health Organization. Geneva, pages 208. Journal of Food Science and Technology, 47(4), 465467. https://doi.org/10.1007/s13197-0100079-0

9. Kariko K, Muramatsu H, Welsh FA, Ludwig J, Kato $\mathrm{H}$, Akira $\mathrm{S}$, et al. Incorporation of pseudouridine into mRNA yields superior nonimmunogenic vector with increased translational capacity and biological stability. MolTher.2008;16:183340. doi: $10.1038 / \mathrm{mt} .2008 .200$

10. Kew, O. M., Sutter, R. W., de Gourville, E. M., Dowdle, W. R., \& Pallansch, M. A. 
(2005). Vaccine-Derived Polioviruses And The Endgame Strategy For Global Polio Eradication. Annual Review of Microbiology, 59(1), 587635. https://doi.org/10.1146/annurev.micro.58.03 0603.123625

11. Li, L., \& Petrovsky, N. (2015). Molecular mechanisms for enhanced DNA vaccine immunogenicity. Expert Review of Vaccines, 15(3), 313-329. https://doi.org/10.1586/14760584.2016.112 4762

12. Mayer KH, Seaton KE, Huang $\mathrm{Y}$, Grunenberg N, Isaacs A, Allen $\mathrm{M}$, et al. Safety, pharmacokinetics, and immunological activities of multiple intravenous or subcutaneous doses of an anti-HIV monoclonal antibody, VRC01, administered to HIV-uninfected adults: results of a phase 1 randomized trial. PLoS Med.2017; 14:e1002435. doi: 10.1371/journal.pmed.1002435

13. Minor, P. D. 1993. Attenuated poliovirus; attenuation by $5^{\prime}$-noncoding region sitedirected mutagenesis for use as a recombinant vaccine. (1993). Vaccine, 11(6), 687.https://doi.org/10.1016/0264410x(93)90321-n

14. Orenstein WA, Bernier RH, Dondero TJ, Hinman AR, Markes JS, Bart KJ, et al. Assessing Vaccine Efficacy In The Field. Epidemiologic Reviews, 1988 ;10(1), 212241.

https://doi.org/10.1093/oxfordjournals.epire v.a036023

15. Parker R, Song H. The enzymes and control of eukaryotic mRNA turnover. Nat Struct Mol Biol. (2004) 11:121-7. doi: $10.1038 / \mathrm{nsmb} 724$

16. Plotkin S. History of vaccination. Proc Natl Acad Sci USA. 2014; 111:12283-7. doi: 10.1073/pnas.1400472111

17. Ramanathan A, Robb GB, Chan SH. mRNA capping: biological functions and applications. Nucleic Acids Res.2016; 44:7511-26. doi: 10.1093/nar/gkw551

18. Rappuoli R, Bottomley MJ, D'Oro U, Finco $\mathrm{O}$, De Gregorio E. Reverse vaccinology 2.0: human immunology instructs vaccine antigen design. J Exp Med. 2016; 213:46981. doi: $10.1084 /$ jem. 20151960

19. Rappuoli R. Reverse vaccinology. Curr Opin Microbiol.2000;3:445-50. doi: 10.1016/S1369-5274(00)00119-3
20. Sok D, Burton DR. HIV Broadly neutralizing antibodies: taking good care of the 98. Immunity. (2016) 45:958-60. doi: 10.1016/j.immuni.2016.10.033

21. The cap and $\operatorname{poly}(\mathrm{A})$ tail function synergistically to regulate mRNA translational efficiency. Genes Dev.1991; 5:2108-16. doi: 10.1101/gad.5.11.2108

22. Thess A, Grund S, Mui BL, Hope MJ, Baumhof $\mathrm{P}$, Fotin-Mleczek $\mathrm{M}$, et al. Sequence-engineered mRNA without chemical nucleoside modifications enables an effective protein therapy in large animals.Mol Ther.2015;23:1456-64. doi: $10.1038 / \mathrm{mt} .2015 .103$

23. U.S Department of Health and Human Services. Vaccine product approval process. U.S. Food and Drug Administration. Updated 01/09/2018. Geller, J. (2018). The Food and Drug Administration Approves More Than a Dozen Devices Through the Premarket Approval Application Process. Journal of Clinical Engineering, 43(4), 131137.

https://doi.org/10.1097/jce.00000000000002 91

24. U.S. Department of Health and Human Services. Investigational New Drug (IND) Application. U.S. Food and Drug Administration. Updated 10/05/2017. FDA Expands List of "Do Not Compound" Drug Products. (2017). Journal of Pain \& Palliative Care Pharmacotherapy, 31(1), 7678.

https://doi.org/10.1080/15360288.2016.127 6996rug Administration.

25. Vernikos G, Medini D. Bexsero® chronicle. Pathog Global Health. 2014; 108:305-16. doi: 10.1179/2047773214Y.0000000162

26. Vivinus S, Baulande S, Van Zanten $M$, Campbell F, Topley P, Ellis JH, et al. An element within the $5^{\prime}$ untranslated region of human Hsp70 mRNA which acts as a general enhancer of mRNA translation. Eur J Biochem.2001;268:1908-17. doi: 10.1046/j.1432-1327.2001.0064.x

27. Weinberg, G. A., \& Szilagyi, P. G. Vaccine Epidemiology: Efficacy, Effectiveness, and the Translational Research Roadmap. The Journal of Infectious Diseases, (2010). 201(11), 1607-1610. https://doi.org/10.1086/652404

28. Weissman D, Pardi N, Muramatsu $H$, Kariko K. HPLC purification of in vitro transcribed long RNA. Methods Mol Biol. 
(2013) 969:43-54. doi: 10.1007/978-162703-260-5_3

29. WHO (2017), Consolidated Guidelines on the use of antiretroviral drugs for treating and preventing HIV infection include recommendations on routine monitoring "What's new in treatment monitoring viral load and CD4 testing(2017).

30. WHO Guidelines for Assuring the Quality of DNA Vaccines. (1998). Biologicals, 26(3), 205-212. https://doi.org/10.1006/biol.1998.0155

31. Williams, J. (2013). Vector Design for Improved DNA Vaccine Efficacy, Safety and Production. Vaccines, 1(3), 225-249. https://doi.org/10.3390/vaccines1030225

32. World Health Organization. State of the World's Vaccines and Immunization. World Health Organization (WHO), UNICEF, World Bank.2020:5-210.
33. Zhu W, Zhong Z, Chen CY. Concerted action of poly(A) nucleases and decapping enzyme in mammalian mRNA turnover. Nat Struct Mol Biol.2005; 12:1054-63. doi: 10.1038/nsmb1016

34. Zohra, F. T., Chowdhury, E. H., Tada, S., Hoshiba, T., \& Akaike, T. (2007). Effective delivery with enhanced translational activity synergistically accelerates mRNA-based transfection. Biochemical and Biophysical Research Communications, 358(1), 373378.

https://doi.org/10.1016/j.bbrc.2007.04.059

How to cite this article: Kumari S. Exigent encounters with vaccines perplexes. Int $J$ Health Sci Res. 2021; 11(7): 322-338. DOI: https://doi. org/10.52403/ijhsr.20210745 